\section{Photo-Induced Atom-Transfer Radical Reactions Using Charge-Transfer Complex between Iodine and Tertiary Amine}

\author{
Eito Yoshioka, Shigeru Kohtani, Takurou Hashimoto, \\ Tomoko Takebe, and Hideto Miyabe*
}

School of Pharmacy, Hyogo University of Health Sciences; 1-3-6 Minatojima, Chuo-ku, Kobe 650-8530, Japan.

Received October 17, 2016; accepted October 24, 2016

In the presence of charge-transfer complexes between iodine and tertiary amines, the aqueous-medium atom-transfer radical reactions proceeded under visible light irradiation without the typical photocatalysts.

Key words radical; iodine; photochemistry; perfluoroalkyl; charge-transfer complex; aqueous media

The charge-transfer complex (CT complex) is formed by weak association of electron-donor and electron-acceptor. Particularly, the CT complexes between metal atoms and ligands are widely studied in inorganic chemistry. ${ }^{1,2)}$ In recent years, the metal-to-ligand charge transfer in transition metal photocatalysts has been applied to the synthetic organic chemistry. $^{3-5)}$ Although the physical properties of organic CT complexes are investigated, ${ }^{6-10)}$ less is known about the utility of organic CT complexes in synthetic reactions. ${ }^{11-15)}$ In our studies on the radical reactions using $\mathrm{Ru}$-catalyst or rhodamine $\mathrm{B}$ as a photocatalyst, ${ }^{16-19)}$ we found that the some reactions proceeded in the absence of these photocatalysts. Therefore, our laboratory is interested in developing a new method which doesn't require the external photocatalysts. In this communication, we report the experiments to prove the utility of organic CT complexes between iodine $\left(\mathrm{I}_{2}\right)$ and tertiary amines in the aqueous-medium carbon-carbon bond-forming radical reactions.

Iodine is known to interact with amines to form the CT complexes, which have the two broader absorption bands at ca. 230-280 and 410-430 $\mathrm{nm}^{20,21)}$ (Chart 1). Therefore, we expected that the visible light irradiation of CT complex $\mathbf{A}$ in the ground state gives the excited state $\mathbf{B}$, which may promote the single electron transfer (SET) from the donor amine to the acceptor iodine giving the iodine radical. ${ }^{22-24)}$

At first, we studied the effect of CT complexes derived from $\mathrm{I}_{2}$ and several amines on the iodine atom-transfer radical reaction of alkene 1 with $i-\mathrm{C}_{3} \mathrm{~F}_{7} \mathrm{I}$ (Table 1). In the presence of $\mathrm{I}_{2}(0.1 \mathrm{eq})$ and trimethylamine $(1.1 \mathrm{eq})$, the biphasic solution of
1 and $i-\mathrm{C}_{3} \mathrm{~F}_{7} \mathrm{I}(5 \mathrm{eq})$ in $\mathrm{H}_{2} \mathrm{O}$ was stirred for $1 \mathrm{~h}$ with white LED light $(400-700 \mathrm{~nm}, 1000 \mathrm{~lm})$ irradiation under Ar atmosphere (entry 1). In this reaction, $1.1 \mathrm{eq}$ of trimethylamine were employed, because $1.0 \mathrm{eq}$ of amine also acts as an electron-donor leading to C. As expected, the desired product $\mathbf{2}$ was obtained in $89 \%$ yield. In marked contrast, the reaction did not occur when MeCN was employed as solvent (entry 2). The use of $(i-\mathrm{Pr})_{2} \mathrm{NEt}$ as a tertiary amine led to enhancement in chemical yield (entry 3), although pyridine did not promote the reaction probably due to its low reactivity as an electron-donor (entry 4). The chemical yield of $\mathbf{2}$ dramatically decreased by using secondary amine such as $(i-\mathrm{Pr})_{2} \mathrm{NH}$, owing to the oxidation of secondary amine by $\mathrm{I}_{2}$ (entry 5). ${ }^{25-27)}$ Interestingly, in the absence of iodine, this transformation took place slowly by using $(i \text {-Pr })_{2} \mathrm{NEt}$ (entry 6). Even in the absence of $\mathrm{I}_{2}, \mathrm{CT}$ complex between $(i-\mathrm{Pr})_{2} \mathrm{NEt}$ and $\mathrm{I}_{2}$ would be formed, because $i-\mathrm{C}_{3} \mathrm{~F}_{7} \mathrm{I}$ is gradually decomposed to give $\mathrm{I}_{2}$. However, in the absence of amine, the reaction using only iodine did not occur and the solubility of iodine also decreased without the association with amine (entry 7). Therefore, this CT complex-promoted reaction is differentiated from the reported iodine-mediated radical reactions. ${ }^{28,29)}$ Theoretical and computational studies

Table 1. Iodine-Atom Transfer Reaction Using Charge-Transfer Complexes $^{a)}$

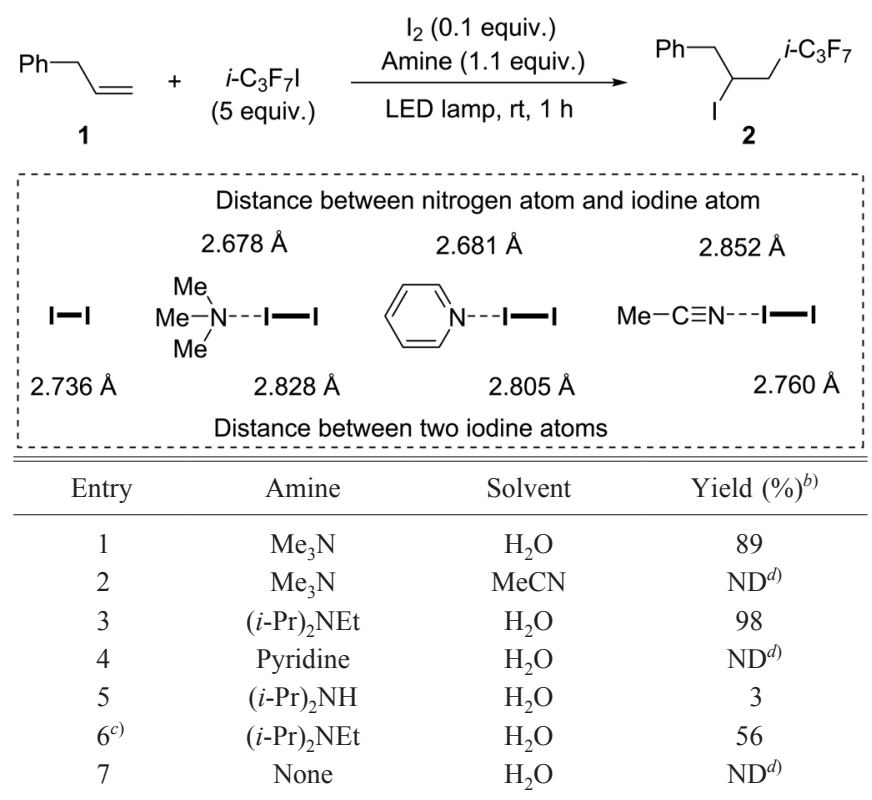

a) Reactions of 1 (1 eq) with $i-\mathrm{C}_{3} \mathrm{~F}_{7} \mathrm{I}$ (5 eq) were carried out in the presence of $\mathrm{I}_{2}(0.1 \mathrm{eq})$ and amine (1.1 eq) under the LED light irradiation. The calculation studies were performed on density functional B3LYP $6-311+\mathrm{G}^{* *}$ by using Spartan'10 (WAVEFUNCTION, INC.). b) Isolated yields. c) Reaction was carried out in the absence of $\mathrm{I}_{2}$ for $4 \mathrm{~h} . d$ ) The formation of product 2 was not detected.

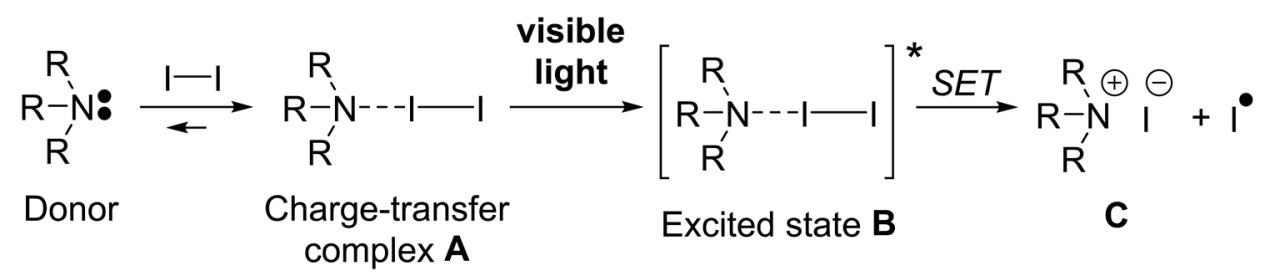

Chart 1. Charge-Transfer Complex between Amine and Iodine 
Table 2. Reaction of 3-8 with $i-\mathrm{C}_{3} \mathrm{~F}_{7}{ }^{a}{ }^{a}$

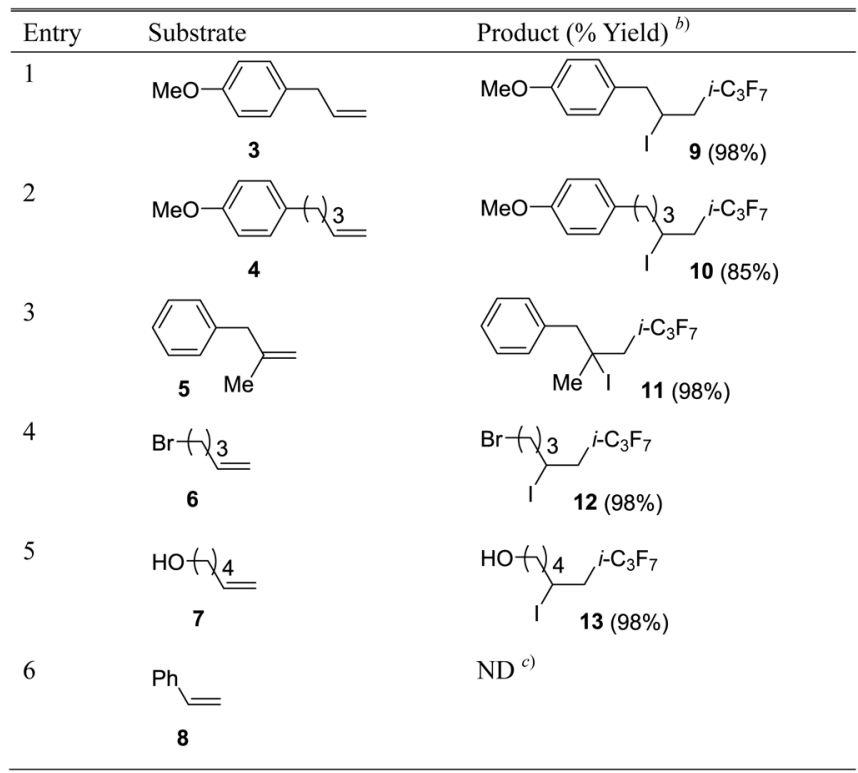

a) Reactions of 3-8 ( $1 \mathrm{eq})$ with $i-\mathrm{C}_{3} \mathrm{~F}_{7} \mathrm{I}(5 \mathrm{eq})$ were carried out in $\mathrm{H}_{2} \mathrm{O}$ in the presence of $\mathrm{I}_{2}(0.1 \mathrm{eq})$ and $(i-\mathrm{Pr})_{2} \mathrm{NEt}(1.1 \mathrm{eq})$ for $1 \mathrm{~h}$ under the LED light irradiation. $\left.b\right)$ Isolated yields. $c$ ) The formation of product was not detected.
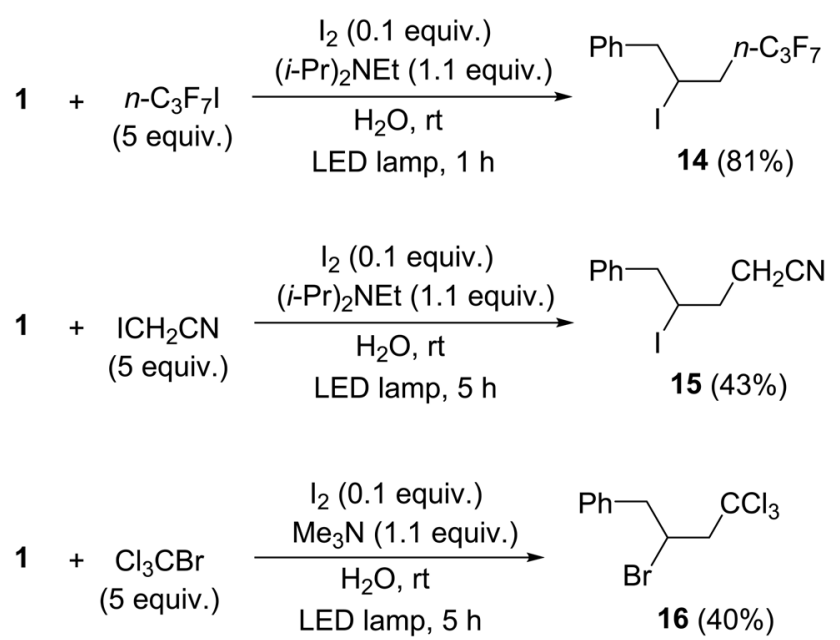

Chart 2. Reaction with Other Carbon Radical Precursors

on halogen bonding interactions have been a subject of current interest. $^{30,31)}$ To understand the above results, we calculated the optimized structures of iodine complexes. The calculation shows that the noncovalent interaction between trimethylamine and $\mathrm{I}_{2}$ is strong to form the stable CT complex, while pyridine weakly interacts with $\mathrm{I}_{2}$. Additionally, we presume that the negligible interaction of $\mathrm{MeCN}$ solvent with $\mathrm{I}_{2}$ may suppress the formation of CT complex.

We next explored the iodine atom-transfer radical reaction of various alkenes $3-\mathbf{8}$ with $i-\mathrm{C}_{3} \mathrm{~F}_{7} \mathrm{I}$ under the optimized reaction conditions (Table 2). Except for styrene 8, alkenes 3-7 reacted with excellent chemical efficiencies and regioselectivities. It is important to note that the reactions of alkene 6 having bromine atom and alkene 7 having hydroxy group proceeded without any problems.

To study the viability of the present method, $n-\mathrm{C}_{3} \mathrm{~F}_{7} \mathrm{I}$, $\mathrm{ICH}_{2} \mathrm{CN}$ and $\mathrm{CCl}_{3} \mathrm{Br}$ were next employed as carbon radical
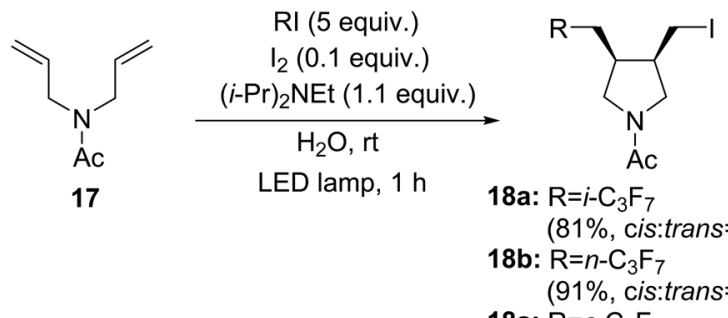

$(81 \%$, cis:trans $=75: 25)$

18b: $\mathrm{R}=n-\mathrm{C}_{3} \mathrm{~F}_{7}$

$(91 \%$, cis:trans $=72: 28)$

18a: $\mathrm{R}=c-\mathrm{C}_{6} \mathrm{~F}_{11}$

$(89 \%$, cis:trans $=70: 30)$
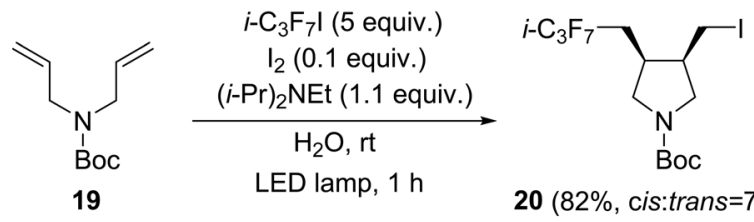

$20(82 \%$, cis:trans $=76: 24)$
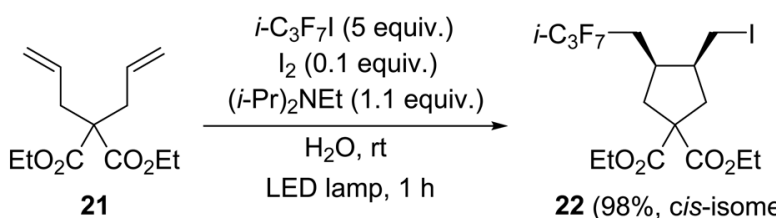

22 (98\%, cis-isomer)

Chart 3. Cascade Radical Addition-Cyclization-Trapping Reaction

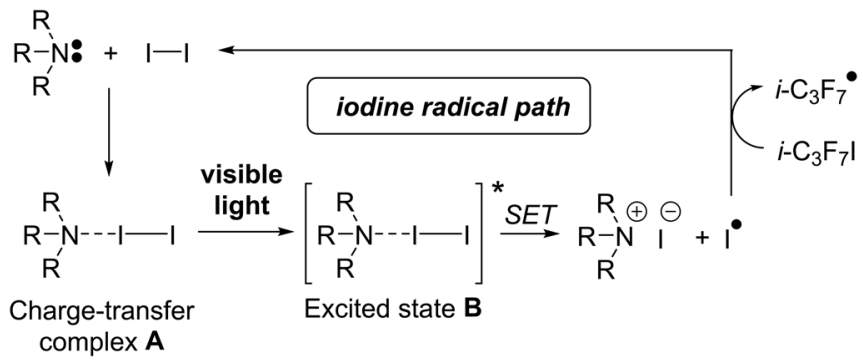

Chart 4. Possible Reaction Pathway

precursors (Chart 2). The reaction of 1 with $n-\mathrm{C}_{3} \mathrm{~F}_{7} \mathrm{I}$ proceeded to give the product $\mathbf{1 4}$ in $81 \%$ yield. Although the reaction in $\mathrm{MeCN}$ did not occur (entry 2 in Table 1), $\mathrm{ICH}_{2} \mathrm{CN}$ having cyano group worked as a radical precursor to give the adduct 15 in 43\% yield. Moreover, the bromine atom-transfer radical reaction using $\mathrm{CCl}_{3} \mathrm{Br}$ took place with moderate chemical efficiency.

We finally investigated the radical addition-cyclizationtrapping reaction of symmetrical substrates 17, 19 and 21 in aqueous media (Chart 3). When $i-\mathrm{C}_{3} \mathrm{~F}_{7} \mathrm{I}$ was employed, the reaction of 17 was completed within $1 \mathrm{~h}$ to give the products cis-18a and trans-18a in $81 \%$ combined yield. Other radical precursors $n-\mathrm{C}_{3} \mathrm{~F}_{7} \mathrm{I}$ and $c-\mathrm{C}_{6} \mathrm{~F}_{11} \mathrm{I}$ worked well. Similarly, the reaction of 19 with $i-\mathrm{C}_{3} \mathrm{~F}_{7} \mathrm{I}$ gave the products cis-20 and trans$\mathbf{2 0}$ in $82 \%$ combined yield. The reaction of $\mathbf{2 1}$ also proceeded effectively to give the product $\mathbf{2 2}$ in $98 \%$ yield with excellent cis-diastereoselectivity.

The possible reaction pathway for the generation of $i-\mathrm{C}_{3} \mathrm{~F}_{7}$ radical is shown in Chart 4 . The reaction is initiated by the visible light irradiation of CT complex $\mathbf{A}$ in the ground state to produce the excited state $\mathbf{B}$. We presume the generation of an iodine radical via the single electron transfer from the donor amine to the acceptor iodine in the excited state $\mathbf{B}$, which is evident from observation of the transient species 
generated in the photoexcitation of quinuclidine- $\mathrm{I}_{2}$ and triethylenediamine $-\mathrm{I}_{2}$ complexes by means of transient absorption spectroscopy, ${ }^{22)}$ although it cannot be excluded that the excited state $\mathbf{B}$ acts as a reducing agent toward $i-\mathrm{C}_{3} \mathrm{~F}_{7} \mathrm{I}$. Finally, an iodine radical reacts with $i-\mathrm{C}_{3} \mathrm{~F}_{7} \mathrm{I}$ to give $i-\mathrm{C}_{3} \mathrm{~F}_{7}$ radical and the regenerated iodine.

In summary, we have demonstrated that $\mathrm{CT}$ complexes between iodine and tertiary amines have the potential to induce the atom-transfer radical reactions in aqueous media. In addition to typical photocatalysts, the CT complexes disclosed a broader utility of photo-induced radical reactions.

Acknowledgment This work was supported by JSPS KAKENHI Grant-in-Aid for Scientific Research (C) Grant Number 16K08188 (to H.M.).

Conflict of Interest The authors declare no conflict of interest.

\section{References}

1) Akimov A. V., Neukirch A. J., Prezhdo O. V., Chem. Rev., 113, 4496-4565 (2013).

2) Rondi A., Rodriguez Y., Feurer T., Cannizzo A., Acc. Chem. Res., 48, 1432-1440 (2015).

3) Tucker J. W., Stephenson C. R. J., J. Org. Chem., 77, 1617-1622 (2012).

4) Ischay M. A., Yoon T. P., Eur. J. Org. Chem., 2012, 3359-3372 (2012).

5) Shi L., Xia W., Chem. Soc. Rev., 41, 7687-7697 (2012).

6) Onda K., Yamochi H., Koshihara S., Acc. Chem. Res., 47, 3494 3503 (2014)

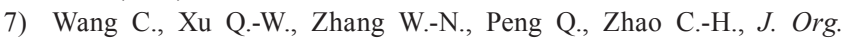
Chem., 80, 10914-10924 (2015)

8) Geng Y., Pfattner R., Campos A., Hauser J., Laukhin V., Puigdollers J., Veciana J., Mas-Torrent M., Rovira C., Decurtins S., Liu S., Chem. Eur. J., 20, 7136-7143 (2014).

9) Haberhauer G., Gleiter R., Burkhart C., Chem. Eur. J., 22, 971-978 (2016).
10) Rosokha S. V., Kochi J. K., Acc. Chem. Soc., 41, 641-653 (2008).

11) Koshima H., Ding K., Chisaka Y., Matsuura T., Ohashi Y., Mukasa M., J. Org. Chem., 61, 2352-2357 (1996).

12) González-Béjar M., Stiriba S.-E., Miranda M. A., Pérez-Prieto J., Org. Lett., 9, 453-456 (2007).

13) Arceo E., Jurberg I. D., Alvarez-Fernández A., Melchiorre P., Nat. Chem., 5, 750-756 (2013).

14) Kandukuri S. R., Bahamonde A., Chatterjee I., Jurberg I. D., Escudero-Adán E. C., Melchiorre P., Angew. Chem. Int. Ed., 54, 1485-1489 (2015).

15) Woźniak Ł., Murphy J. J., Melchiorre P., J. Am. Chem. Soc., 137, 5678-5681 (2015).

16) Yoshioka E., Kohtani S., Tanaka E., Miyabe H., Synlett, 24, 1578 1582 (2013).

17) Yoshioka E., Kohtani S., Tanaka E., Hata Y., Miyabe H., Tetrahedron, 71, 773-781 (2015).

18) Yoshioka E., Kohtani S., Jichu T., Fukazawa T., Nagai T., Takemoto Y., Miyabe H., Synlett, 26, 265-270 (2015).

19) Yoshioka E., Kohtani S., Jichu T., Fukazawa T., Nagai T., Kawashima A., Takemoto Y., Miyabe H., J. Org. Chem., 81, 7217-7229 (2016).

20) Nagakura S., J. Am. Chem. Soc., 80, 520-524 (1958).

21) Yada H., Tanaka J., Nagakura S., Bull. Chem. Soc. Jpn., 33, 1660 1667 (1960).

22) Halpern A. M., Weiss K., J. Phys. Chem., 72, 3863-3870 (1968).

23) Lenderink E., Duppen K., Everdij F. P. X., Mavri J., Torre R., Wiersma D. A., J. Phys. Chem., 100, 7822-7831 (1996).

24) DeBoer G., Burnett J. W., Fujimoto A., Young M. A., J. Phys. Chem., 100, 14882-14891 (1996).

25) Ishihara M., Togo H., Synlett, 2006, 227-230 (2006).

26) Shie J.-J., Fang J.-M., J. Org. Chem., 72, 3141-3144 (2007).

27) Lin C., Lai P.-T., Liao S. K.-S., Hung W.-T., Yang W.-B., Fang J.-M., J. Org. Chem., 73, 3848-3853 (2008).

28) Usami K., Nagasawa Y., Yamaguchi E., Tada N., Itoh A., Org. Lett., 18, 8-11 (2016)

29) Lin Y., Lu G., Cai C., Yi W., Org. Lett., 17, 3310-3313 (2015).

30) Kolář M. H., Hobza P., Chem. Rev., 116, 5155-5187 (2016).

31) Tsuzuki S., Wakisaka A., Ono T., Sonoda T., Chem. Eur. J., 18, 951-960 (2012). 